\title{
A Fractional Order Hepatitis C Mathematical Model with Mittag- Leffler Kernel
}

\author{
Hashim M. Alshehri $\mathbb{D D}^{1}$ and Aziz Khan ${ }^{2}$ \\ ${ }^{1}$ Department of Mathematics, Faculty of Science, King Abdulaziz University, Jeddah 21521, Saudi Arabia \\ ${ }^{2}$ Department of Mathematics and General Sciences, Prince Sultan University, Riyadh, Saudi Arabia \\ Correspondence should be addressed to Hashim M. Alshehri; hmalshehri@kau.edu.sa
}

Received 27 June 2021; Revised 1 August 2021; Accepted 28 September 2021; Published 26 October 2021

Academic Editor: Anita Tomar

Copyright (c) 2021 Hashim M. Alshehri and Aziz Khan. This is an open access article distributed under the Creative Commons Attribution License, which permits unrestricted use, distribution, and reproduction in any medium, provided the original work is properly cited.

In this paper, a mathematical fractional order Hepatitis $\mathrm{C}$ virus (HCV) spread model is presented for an analytical and numerical study. The model is a fractional order extension of the classical model. The paper includes the existence, singularity, Hyers-Ulam stability, and numerical solutions. Our numerical results are based on the Lagrange polynomial interpolation. We observe that the model of fractional order has the same behavior of the solutions as the integer order existing model.

\section{Introduction}

Hepatitis $\mathrm{C}$ is a kind of viral maladies caused by the Hepatitis $\mathrm{C}$ virus (HCV), which mostly damages the liver. People generally have minor or no symptoms when they first become infected. Black urine, Fever, yellow-tinged skin, and abdominal pain are all symptoms that might occur. The virus remains in the liver, in roughly 75 percent to 85 percent of patients who are infected. Initially, in the period of a chronic infection, there are usually no symptoms. However, it frequently develops to cirrhosis over time. Hepatitis $\mathrm{C}$, on the other hand, can sometimes lead to liver cancer, blood cancer, and liver failure [1]. The most common way for $\mathrm{HCV}$ to spread is by blood-to-blood contact, which is related to injectable usage of drugs, improperly cleaned equipment for medical care, needle stick injuries in health care, and transfusions. The most typical reason for liver transplantation is Hepatitis $C$, even though virus generally returns after the procedure. Hepatitis $C$ infects an estimated 71 million people (1 percent of the global population) in 2015. Low- and middle-income nations bear the brunt of the health burden, with Africa and Central and East Asia having the greatest rates of prevalence. In 2015, Hepatitis C caused around 167,000 liver cancer deaths and 326,000 cirrhosis deaths. 15th Hepatitis C is a disease that can be transmitted from one person to another. Hepatitis $\mathrm{C}$ was first identified in the 1970s, it was thought to be a kind of non-A non-B Hepatitis, and its presence was confirmed in 1989. Only humans and chimps are infected with Hepatitis C; for more details, see $[2,3]$.

In natural and physical sciences, mathematical and computational tools have been used to investigate phenomena at many scales, ranging from the global human population to individual atoms within a biomolecule. The relevant modeling methodologies span time spans ranging from years to picoseconds, region to region of interest (impacts ranging from evolutionary to atomic), and importance. This exploration will go over some of the most common and useful approaches in mathematics and computing. Differential equations, statistical models, dynamical systems, and game theoretic models are all examples of mathematical models; we refer to [4-10]. These and other types of models can be mixed and matched, resulting in a single model that has a diverse set of abstract structures. Logic models can be used in mathematical models in general. In many instances, the quality of a scientific topic is determined by how well theoretical mathematical models accord with the results of repeated experiments. As better theories are discovered, a lack of concordance between mathematical models that are theoretical and experimental findings frequently leads to 
significant advancements. Many mathematical models were formulated for the Hepatitis $\mathrm{C}$ diseases to understand the dynamics of the diseases and control the spreading of diseases; we refer to [11-14].

Fractional calculus is a discipline of mathematics that investigates the various ways in which the differentiation operator can be defined in terms of real or complex number powers. Because of their ability to include notion of nonlocal operators used to incorporate more complicated natural phenomena into mathematical equations, differential equations have attracted many scholars from practically all disciplines of science, technology, and engineering in recent years. The exponential decay law, the power law, and the extended Mittag-Leffler law were recommended as three dominants in fractional calculus. The kernel Mittag-Leffler function was shown to be more broadly applicable than the power law and exponential decay functions; both Riemann-Liouville and Caputo-Fabrizio are special examples of the Atangana-Baleanu fractional operator; we refer to [15-18]. Many biological models have been studied on fractional operators; Atangana and Alqahtani [19] considered a mathematical model river blindness as in Caputo sense and beta operators. Stability and numerical solutions were obtained for the fractional order model. GómezAguilar et al. [20] examined a cancer model in three dimensions in the sense of Caputo-Fabrizio-Caputo and the novel fractional derivative with Mittag-Leffler kernel. Special solutions were obtained by an iterative process that used the Laplace transform rule, Sumudu-Picard integration approach, and the Adams-Moulton approach. Shah and Bushnaq [21] evaluated an endemic infection model in the fractional sense. Numerical solutions were obtained for the proposed model by combining the Laplace transform with the Adomian decomposition approach. Arfan et al. [22] studied semianalytical solutions for a fractional order COVID-19 model under Caputo derivative; for more details, see [23-26].

Inspired from the above literature, in this paper, we consider a Hepatitis $\mathrm{C}$ model in the sense of a fractional order derivative. Furthermore, we investigate the existence and uniqueness of the fractional order model with the help of a fixed point theorem and stability analysis of the fractional order Hepatitis C model. Finally, numerical simulations of the solutions are demonstrated and compared with classical derivatives by using different values of fractional order and parameters. This paper is organized as follows; Section 1: introduction of the paper; Section 2: framework of the model; Section 3: preliminaries; Section 4: existence of solutions; Section 5: numerical data fitting; Section 6: conclusion.

\section{Framework of the Model}

In this section, we discuss an integer order Hepatitis C model. The population is split into four classes based on their size. $\mathbb{S}$ denotes class of susceptible, $\mathbb{\square}$ class of acutely infected, $\mathbb{P}$ class of persistently infected, and $\mathbb{T}$ class of treatment for infection:

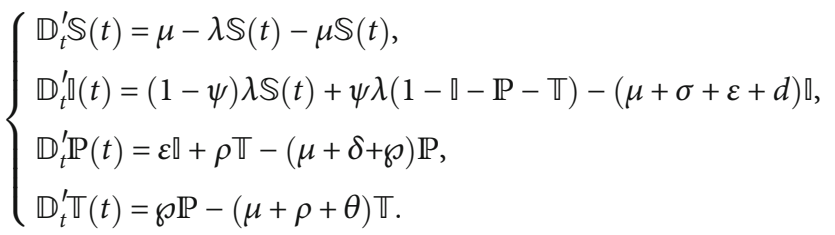

With the initial conditions

$$
\begin{aligned}
& \mathbb{S}(0) \geq 0, \\
& \mathbb{}(0) \geq 0, \\
& \mathbb{P}(0) \geq 0, \\
& \mathbb{T}(0) \geq 0,
\end{aligned}
$$

where $\mu$ is the rate of natural death, $\lambda$ is force of infection, $\psi$ is the rate of susceptibility of recovered, $\sigma$ is the rate of recovery from acute infection, $\varepsilon$ is the rate of progression to chronic infection, $d$ is the rate of death due to acute infection, $\rho$ is the rate of treatment failure of chronically infected, $\delta$ is the rate of recovery from chronic infection, $\wp$ is the rate of treatment of chronically infected, and $\theta$ is the rate of treatment cure. For more details on the existence of infection, endemic equilibrium, reproduction numbers, and stability of endemic equilibrium, see [11-13].

Definition 1 (see [17, 27]). On the basis of Mittag-Leffler kernel and $\psi \in H^{*}(a, b), b>a$, for $\mathrm{Q} \in[0,1]$, the $A B C$-fractional differential operator is given as

$$
{ }_{a}^{A B C} \mathbb{D}_{\wp}^{\mathrm{\varrho}} \psi(\wp)=\frac{\beta(\mathrm{\varrho})}{1-\mathrm{\varrho}} \int_{a}^{\wp} \psi^{\prime}(s) E_{\mathrm{\varrho}}\left[\frac{-\mathrm{\varrho}(\wp-s)^{\varrho}}{1-\varrho}\right] d s,
$$

where $B(\mathrm{Q})$ denote a weighted function which satisfied the main property $\beta(0)=\beta(1)=1$ :

$$
E_{\rho}(h)=\sum_{r=0}^{\infty} \frac{h^{r}}{(1+\rho r)}, \quad \rho>0 .
$$

Definition 2 (see [17, 27]). On the basis of Mittag-Leffler kernel and $\psi \in H^{*}(a, b), b>a, \varrho \in[0,1]$, the $A B R$-fractional derivative is defined as

$$
{ }_{a}^{A B R} \mathbb{D}_{\wp}^{\mathrm{\varrho}} \psi(\wp)=\frac{\beta(\mathrm{\varrho})}{1-\varrho} \frac{d}{d \wp} \int_{a}^{\wp} \psi(s) E_{\mathrm{\varrho}}\left[\frac{-\mathrm{\varrho}(\wp-s)^{\varrho}}{1-\varrho}\right] d s .
$$

Definition 3 (see [17, 27]). Let $\psi \in H^{*}(a, b), b>a, 0<\rho<1$; the $A B$-integral is given

$$
{ }_{a}^{A B} \square_{\wp} \psi(\wp)=\frac{1-\mathrm{\varrho}}{\beta(\mathrm{\varrho})} \psi(\wp)+\frac{\mathrm{\varrho}}{\beta(\mathrm{\varrho}) \Gamma(\mathrm{\varrho})} \int_{a}^{\wp} \psi(s)(\wp-s)^{\mathrm{Q}-1} d s .
$$

Lemma 4 (see [17]). Let function $\psi$, then the $A B$ fractional integral and derivative satisfy the following special character of Newton-Leibniz formula: 


$$
{ }_{a}^{A B} \mathbb{Q}_{\wp}^{\varrho}\left({ }_{a}^{A B C} \mathbb{D}_{\wp}^{\varrho} \psi(\wp)\right)=\psi(\wp)-\psi(a)
$$

\section{Existence Criteria}

Let $\mathscr{B}=\mathscr{I} \times \mathbb{R}^{4} \longrightarrow \mathbb{R}$, where $\mathscr{I}=[0, T]$, for $0<t<T<\infty$, with a norm defined by $\|(\mathbb{S}, \mathbb{\mathbb { N }}, \mathbb{P}, \mathbb{\mathbb { T }})\|=\max _{t \in \mathscr{I}}\{|\mathbb{S}|+|\mathbb{\mathbb { q }}|+\mid$ $\mathbb{P}|+| \mathbb{T} \mid\}$. Then, clearly, $(\mathscr{B},\|\cdot\|)$ is Banach's space. Let us consider system (1) in the sense of fractional order operator:

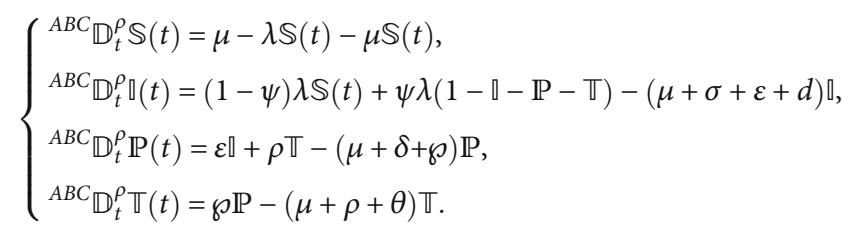

By employing Definition (3) to (8), we have

$$
\begin{aligned}
& \mathbb{S}(t)-\mathbb{S}(0)=\frac{1-\varrho}{\beta(\varrho)}(\mu-\lambda \mathbb{S}-\mu \mathbb{S}(t)) \\
& +\frac{\mathrm{\varrho}}{\beta(\mathrm{\varrho}) \Gamma(\mathrm{\varrho})} \int_{0}^{t}(t-s)^{\mathrm{Q}-1}(\mu-\lambda \mathbb{S}-\mu \mathbb{S}) d s, \\
& \llbracket(t)-\llbracket(0)=\frac{1-\varrho}{\beta(\varrho)}((1-\psi) \lambda \mathbb{S}(t)+\psi \lambda(1-I-\mathbb{P}-\mathbb{T}) \\
& -(\mu+\sigma+\varepsilon+d) \square)+\frac{\mathrm{Q}}{\beta(\mathrm{Q}) \Gamma(\mathrm{\varrho})} \int_{0}^{t}(t-s)^{\mathrm{Q}-1}((1-\psi) \lambda \mathbb{S}(t) \\
& +\psi \lambda(1-I-\mathbb{P}-\mathbb{T})-(\mu+\sigma+\varepsilon+d) \llbracket) d s,
\end{aligned}
$$

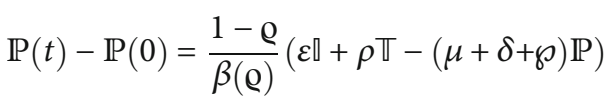

$$
\begin{aligned}
& +\frac{\mathrm{Q}}{\beta(\mathrm{\varrho}) \Gamma(\mathrm{\varrho})} \int_{0}^{t}(t-s)^{\mathrm{Q}-1}(\varepsilon \rrbracket+\rho \mathbb{\mathbb { }}-(\mu+\delta+\wp) \mathbb{P}) d s, \\
& \mathbb{T}(t)-\mathbb{T}(0)=\frac{1-\varrho}{\beta(\varrho)}(\wp \mathbb{P}-(\mu+\rho+\theta) \mathbb{T}) \\
& +\frac{\varrho}{\beta(\varrho) \Gamma(\varrho)} \int_{0}^{t}(t-s)^{\rho-1}(\wp \mathbb{P}-(\mu+\rho+\theta) \mathbb{T}) d s .
\end{aligned}
$$

For simplicity in the above Equation (9), we introduce $\mathbb{H}_{i}$ for $i=1,2,3,4$, given below:

$$
\begin{gathered}
\mathbb{H}_{1}(t, \mathbb{S})=\mu-\lambda \mathbb{S}-\mu \mathbb{S}, \\
\mathbb{H}_{2}(t, \mathbb{\square})=(1-\psi) \lambda \mathbb{S}+\psi \lambda(1-I-\mathbb{P}-\mathbb{T})-(\mu+\sigma+\varepsilon+d) \mathbb{},
\end{gathered}
$$

$$
\begin{gathered}
\mathbb{H}_{3}(t, \mathbb{P})=\varepsilon \rrbracket+\rho \mathbb{\mathbb { V }}-(\mu+\delta+\wp) \mathbb{P}, \\
\mathbb{H}_{4}(t, \mathbb{\mathbb { V }})=\wp \mathbb{P}-(\mu+\rho+\theta) \mathbb{\mathbb { V }},
\end{gathered}
$$

$$
\left\{\begin{array}{l}
\chi_{1}=\lambda-\mu \\
\chi_{2}=\mu+\sigma+\varepsilon+d+\psi \lambda \\
\chi_{3}=(\mu+\delta+\wp) \\
\chi_{4}=(\mu+\rho+\theta)
\end{array}\right.
$$

For proving our results, we consider the following assumption $\mathbb{B}$. For the below continuous functions $\mathbb{S}(t), \mathbb{S}^{*}$ $(t), \square(t), \square^{*}(t), \mathbb{P}(t), \mathbb{P}^{*}(t), \mathbb{T}(t), T^{*}(t), \in L[0,1]$ such that $\| \mathbb{S}$ $(t)\left\|\leq \xi_{1},\right\| \mathbb{\square}(t)\left\|\leq \xi_{2},\right\| \mathbb{P}(t)\left\|\leq \xi_{3}, 0<\right\| \mathbb{T}(t) \| \leq \xi_{4}$, there exist three constant $\kappa_{i}>0, i \in \mathbb{N}_{1}^{3}$, such that the below hold:

$$
\begin{aligned}
\| \mathbb{S}(t \| & \leq \kappa_{1}, \\
\|\mathbb{P}(t)-\mathbb{T}(t)\| & \leq \kappa_{2}, \\
\|\mathbb{P}\| & \leq \kappa_{3} .
\end{aligned}
$$

Theorem 5. $\mathbb{H}_{i}$, for $i \in \mathbb{N}_{1}^{5}$, satisfy Lipschitz condition if $\max \left\{\chi_{i}\right.$, for $\left.i=1,2,3,4\right\}<1$ for $\chi_{i}$ defined in (17).

Consider for $\mathrm{H}_{1}$, below

$$
\begin{aligned}
& \left\|\mathbb{H}_{1}(t, \square)-\mathbb{H}_{1}\left(t, \square^{*}\right)\right\|=\| \mu-\lambda \mathbb{S}(t)-\mu \mathbb{S}(t)-\mu+\lambda \mathbb{S}^{*}(t) \\
& +\mu \mathbb{S}^{*}(t)\|\leq[\lambda-\mu]\| \mathbb{S}-\mathbb{S}^{*}\left\|=\chi_{1}\right\| \mathbb{S}-\mathbb{S}^{*} \| .
\end{aligned}
$$



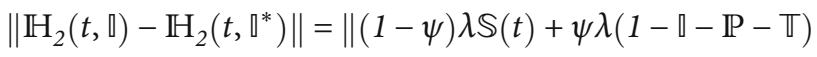

$$
\begin{aligned}
& -(\mu+\sigma+\varepsilon+d) \rrbracket-\left((1-\psi) \lambda \mathbb{S}(t)+\psi \lambda\left(1-\square^{*}-\mathbb{P}-\mathbb{T}\right)\right. \\
& \left.-(\mu+\sigma+\varepsilon+d) \square^{*}\right)\|\leq \psi \lambda\| \square-\square\|+(\mu+\sigma+\varepsilon+d)\| \square-\square^{*} \| \\
& \leq(\mu+\sigma+\varepsilon+d+\psi \lambda)\left\|\square-\square^{*}\right\|=\chi_{2}\left\|\square-\square^{*}\right\| \text {. }
\end{aligned}
$$

$\mathbb{H}_{3}(t, \mathbb{P})$ implies

$$
\begin{aligned}
& \left\|\mathbb{H}_{3}(t, \mathbb{P})-\mathbb{H}_{3}\left(t, \mathbb{P}^{*}\right)\right\|=\| \varepsilon \rrbracket+\rho \mathbb{} \\
& -(\mu+\delta+\wp) \mathbb{P}-\left(\varepsilon \rrbracket+\rho \mathbb{\mathbb { V }}-(\mu+\delta+\wp) \mathbb{P}^{*}\right) \| \\
& \leq(\mu+\delta+\wp)\left\|\mathbb{P}-\mathbb{P}^{*}\right\|=\chi_{3}\left\|\mathbb{P}-\mathbb{P}^{*}\right\| .
\end{aligned}
$$

$\mathrm{H}_{4}(t, \mathbb{T})$ implies

$$
\begin{aligned}
& \left\|\mathbb{H}_{4}(t, \mathbb{T})-\mathbb{T}_{4}\left(t, \mathbb{T}^{*}\right)\right\|=\| \wp \mathbb{P}-(\mu+\rho+\theta) \mathbb{T} \\
& -\left(\wp \mathbb{P}-(\mu+\rho+\theta)-\mathbb{T}^{*}\|\leq(\mu+\rho+\theta)\| \mathbb{T}-\mathbb{T}^{*} \|\right. \\
& \quad=\chi_{4}\left\|\mathbb{T}-\mathbb{T}^{*}\right\| .
\end{aligned}
$$

Thus, from (19)(-)(22), we have that $\mathbb{Q}_{i}$ for $i=1,2,3,4$, satisfying the Lipschitz condition. And this completes the proof. Assuming that $\mathbb{S}(0)=\mathbb{\square}(0)=\mathbb{P}(0)=\mathbb{T}(0)=0$, then we have

$$
\mathbb{S}(t)=\frac{1-\mathrm{\varrho}}{\beta(\mathrm{\varrho})} \mathbb{H}_{1}(t, \mathbb{S}(t))+\frac{\mathrm{\varrho}}{\beta(\mathrm{\varrho}) \Gamma(\mathrm{\varrho})} \int_{0}^{t}(t-s)^{\mathrm{\varrho}-1} \mathbb{H}_{1}(s, \mathbb{S})(s) d s
$$


$\square(t)=\frac{1-\varrho}{\beta(\varrho)} \mathbb{H}_{2}(t, \square(t))+\frac{\varrho}{\beta(\varrho) \Gamma(\varrho)} \int_{0}^{t}(t-s)^{\mathrm{Q}^{-1}} \mathbb{H}_{2}(s, \mathbb{\square})(s) d s$,

$\mathbb{P}(t)=\frac{1-\varrho}{\beta(\varrho)} \mathbb{H}_{3}(t, \mathbb{P}(t))+\frac{\varrho}{\beta(\varrho) \Gamma(\varrho)} \int_{0}^{t}(t-s)^{\rho-1} \mathbb{H}_{3}(s, \mathbb{P})(s) d s$,

$\mathbb{T}(t)=\frac{1-\mathrm{\varrho}}{\beta(\mathrm{\varrho})} \mathbb{H}_{4}(t, \mathbb{T}(t))+\frac{\mathrm{\varrho}}{\beta(\mathrm{\varrho}) \Gamma(\mathrm{\varrho})} \int_{0}^{t}(t-s)^{\mathrm{\varrho}-1} \mathbb{H}_{4}(s, \mathbb{T})(s) d s$

For the iterative scheme of the ABC-fractional order $\mathrm{HCV}$ model (8), define

$$
\begin{aligned}
& \mathbb{S}_{n}(t)=\frac{1-\mathrm{\varrho}}{\beta(\mathrm{\varrho})} \mathbb{H}_{1}\left(t, \mathbb{S}_{n-1}(t)\right)+\frac{\mathrm{\varrho}}{\beta(\mathrm{\varrho}) \Gamma(\mathrm{\varrho})} \int_{0}^{t}(t-s)^{\mathrm{\varrho}-1} \mathbb{H}_{1}\left(s, \mathbb{S}_{n-1}\right)(s) d s, \\
& \mathbb{\square}_{n}(t)=\frac{1-\mathrm{\varrho}}{\beta(\mathrm{\varrho})} \mathbb{H}_{2}\left(t, \mathbb{\square}_{n-1}(t)\right)+\frac{\mathrm{\varrho}}{\beta(\mathrm{\varrho}) \Gamma(\mathrm{\varrho})} \int_{0}^{t}(t-s)^{\mathrm{\varrho}-1} \mathbb{H}_{2}\left(s, \mathbb{\square}_{n-1}\right)(s) d s, \\
& \mathbb{P}_{n}(t)=\frac{1-\mathrm{\varrho}}{\beta(\mathrm{\varrho})} \mathbb{H}_{3}\left(t, \mathbb{P}_{n-1}(t)\right)+\frac{\mathrm{\varrho}}{\beta(\mathrm{\varrho}) \Gamma(\mathrm{\varrho})} \int_{0}^{t}(t-s)^{\mathrm{\varrho}-1} \mathbb{H}_{3}\left(s, \mathbb{P}_{n-1}\right)(s) d s, \\
& \mathbb{T}_{n}(t)=\frac{1-\varrho}{\beta(\varrho)} \mathbb{H}_{4}\left(t, \mathbb{T}_{n-1}(t)\right)+\frac{\varrho}{\beta(\varrho) \Gamma(\varrho)} \int_{0}^{t}(t-s)^{\mathrm{Q}^{-1}} \mathbb{H}_{4}\left(s, \mathbb{T}_{n-1}\right)(s) d s .
\end{aligned}
$$

Theorem 6. The ABC-fractional order HCV model (8) has a solution if

$$
\Delta=\max \left\{\chi_{i}\right\}<1, \quad i \in \mathbb{N}_{1}^{4} .
$$

We define the function

$$
\begin{gathered}
\mathbb{G} 1_{n}(t)=\mathbb{S}_{n+1}(t)-\mathbb{S}(t), \\
\mathbb{G} 2_{n}(t)=\mathbb{\square}_{n+1}(t)-\mathbb{\square}(t), \\
\mathbb{G} 3_{n}(t)=\mathbb{P}_{n+1}(t)-\mathbb{P}(t), \\
\mathbb{G} 4_{n}(t)=\mathbb{T}_{n+1}(t)-\mathbb{T}(t) .
\end{gathered}
$$

By the help of (29) and (30), we have

$$
\begin{aligned}
\left\|\mathbb{G} 1_{n}\right\| \leq & \frac{1-\mathrm{\varrho}}{\beta(\mathrm{\varrho})}\left\|\mathbb{H}_{1}\left(t, \mathbb{S}_{n}(t)\right)-\mathbb{H}_{1}\left(t, \mathbb{S}_{n-1}(t)\right)\right\| \\
& +\frac{\mathrm{\varrho}}{\beta(\mathrm{\varrho}) \Gamma(\mathrm{\varrho})} \int_{0}^{t}(t-s)^{\rho-1} \| \mathbb{H}_{1}\left(s, \mathbb{S}_{n}(s)\right) \\
& -\mathbb{H}_{1}\left(t, \mathbb{S}_{n-1}(t)\right)\left\|d s \leq\left[\frac{1-\mathrm{\varrho}}{\beta(\mathrm{\varrho})}+\frac{1}{\beta(\mathrm{\varrho}) \Gamma(\mathrm{\varrho})}\right] \chi_{1}\right\| \mathbb{S}_{n}-\mathbb{S}_{n-1} \| \\
\leq & {\left[\frac{1-\mathrm{\varrho}}{\beta(\mathrm{\varrho})}+\frac{1}{\beta(\mathrm{\varrho}) \Gamma(\mathrm{\varrho})}\right]^{n} \Delta^{n}\left\|\mathbb{S}_{n}-\mathbb{S}_{n-1}\right\|, }
\end{aligned}
$$

$$
\begin{aligned}
& \left\|\mathbb{G} 2_{n}\right\| \leq \frac{1-\mathrm{\varrho}}{\beta(\mathrm{\varrho})}\left\|\mathbb{H}_{2}\left(t, \mathbb{\square}_{n}(t)\right)-\mathbb{H}_{2}\left(t, \rrbracket_{n-1}(t)\right)\right\| \\
& +\frac{\mathrm{\varrho}}{\beta(\mathrm{\varrho}) \Gamma(\mathrm{\varrho})} \int_{0}^{t}(t-s)^{\rho-1} \| \mathrm{H}_{2}\left(s, \mathbb{n}_{n}(s)\right) \\
& -\mathbb{H}_{2}\left(t, \rrbracket_{n-1}(t)\right)\left\|d s \leq\left[\frac{1-\mathrm{\varrho}}{\beta(\mathrm{\varrho})}+\frac{1}{\beta(\mathrm{\varrho}) \Gamma(\mathrm{\varrho})}\right] \chi_{2}\right\| \mathbb{\square}_{n}-\mathbb{\square}_{n-1} \| \\
& \leq\left[\frac{1-\mathrm{\varrho}}{\beta(\mathrm{\varrho})}+\frac{1}{\beta(\mathrm{\varrho}) \Gamma(\mathrm{\varrho})}\right]^{n} \Delta^{n}\left\|\mathbb{q}_{n}-\mathbb{q}_{n-1}\right\| \text {. }
\end{aligned}
$$

Similarly,

$$
\begin{aligned}
& \left\|\mathbb{G} 3_{n}\right\| \leq \frac{1-\varrho}{\beta(\mathrm{Q})}\left\|\mathbb{H}_{3}\left(t, \mathbb{P}_{n}(t)\right)-\mathbb{H}_{3}\left(t, \mathbb{P}_{n-1}(t)\right)\right\| \\
& +\frac{\mathrm{\varrho}}{\beta(\mathrm{\varrho}) \Gamma(\mathrm{\varrho})} \int_{0}^{t}(t-s)^{\mathrm{Q}-1} \| \mathrm{H}_{3}\left(s, \mathbb{P}_{n}(s)\right) \\
& -\mathbb{H}_{3}\left(t, \mathbb{P}_{n-1}(t)\right)\left\|d s \leq\left[\frac{1-\mathrm{\varrho}}{\beta(\mathrm{\varrho})}+\frac{1}{\beta(\mathrm{\varrho}) \Gamma(\mathrm{\varrho})}\right] \chi_{3}\right\| \mathbb{P}_{n}-\mathbb{P}_{n-1} \| \\
& \leq\left[\frac{1-\mathrm{\varrho}}{\beta(\mathrm{\varrho})}+\frac{1}{\beta(\mathrm{\varrho}) \Gamma(\mathrm{\varrho})}\right]^{n} \Delta^{n}\left\|\mathbb{P}_{n}-\mathbb{P}_{n-1}\right\|, \\
& \left\|\mathbb{G} 4_{n}\right\| \leq \frac{1-\varrho}{\beta(\varrho)}\left\|\mathbb{H}_{4}\left(t, \mathbb{T}_{n}(t)\right)-\mathbb{H}_{4}\left(t, \mathbb{T}_{n-1}(t)\right)\right\| \\
& +\frac{\mathrm{\varrho}}{\beta(\mathrm{\varrho}) \Gamma(\mathrm{\varrho})} \int_{0}^{t}(t-s)^{\rho-1} \| \mathrm{H}_{4}\left(s, \mathbb{\mathbb { T }}_{n}(s)\right) \\
& -\mathbb{H}_{4}\left(t, \mathbb{T}_{n-1}(t)\right)\left\|d s \leq\left[\frac{1-\mathrm{\varrho}}{\beta(\mathrm{\varrho})}+\frac{1}{\beta(\mathrm{\varrho}) \Gamma(\mathrm{\varrho})}\right] \chi_{4}\right\| \mathbb{T}_{n}-\mathbb{T}_{n-1} \| \\
& \leq\left[\frac{1-\varrho}{\beta(\varrho)}+\frac{1}{\beta(\varrho) \Gamma(\varrho)}\right]^{n} \Delta^{n}\left\|\mathbb{T}_{n}-\mathbb{T}_{n-1}\right\|,
\end{aligned}
$$

which ensure that $\mathbb{G}(t)_{n} \longrightarrow 0, i \in \mathbb{N}_{1}^{4}$, as $n \longrightarrow \infty$ for $\Delta<1$, which completes the proof.

\section{Uniqueness Solution}

For our suggested model (8), we study the analysis of the uniqueness of solution.

Theorem 7. The ABC-fractional order Hepatitis C model (8) has a unique solution provided that

$$
\left[\frac{1-\mathrm{\varrho}_{i}}{\beta\left(\mathrm{\varrho}_{i}\right)}+\frac{1}{\beta\left(\mathrm{\varrho}_{i}\right) \Gamma\left(\mathrm{\varrho}_{i}\right)}\right] \chi_{i} \leq 1, \quad i \in \mathbb{N}_{1}^{4}
$$

Assume another solution exist $\overline{\mathbb{S}}(t), \bar{\square}(t), \overline{\mathbb{P}}(t), \overline{\mathbb{T}}(t)$, and $\overline{\mathbb{R}}(t)$ such that 


$$
\begin{aligned}
& \overline{\mathbb{S}}(t)=\frac{1-\mathrm{\varrho}}{\beta(\mathrm{\varrho})} \mathbb{H}_{1}(t, \overline{\mathbb{H}}(t))+\frac{\mathrm{\varrho}}{\beta(\mathrm{\varrho}) \Gamma(\mathrm{\varrho})} \int_{0}^{t}(t-s)^{\mathrm{\varrho}-1} \mathbb{H}_{1}(s, \overline{\mathbb{S}}(s)) d s, \\
& \bar{\square}(t)=\frac{1-\mathrm{\varrho}}{\beta(\mathrm{\varrho})} \mathbb{H}_{2}(t, \bar{\square}(t))+\frac{\mathrm{\varrho}}{\beta(\mathrm{\varrho}) \Gamma(\mathrm{\varrho})} \int_{0}^{t}(t-s)^{\mathrm{\varrho}^{-1}} \mathrm{H}_{2}(s, \bar{\square}(s)) d s, \\
& \overline{\mathbb{P}}(t)=\frac{1-\mathrm{\varrho}}{\beta(\mathrm{\varrho})} \mathbb{H}_{3}(t, \overline{\mathbb{P}}(t))+\frac{\mathrm{\varrho}}{\beta(\mathrm{\varrho}) \Gamma(\mathrm{\varrho})} \int_{0}^{t}(t-s)^{\mathrm{Q}-1} \mathbb{H}_{3}(s, \overline{\mathbb{P}}(s)) d s, \\
& \overline{\mathbb{T}}(t)=\frac{1-\mathrm{\varrho}}{\beta(\mathrm{\varrho})} \mathbb{H}_{4}(t, \overline{\mathbb{T}}(t))+\frac{\mathrm{\varrho}}{\beta(\mathrm{\varrho}) \Gamma(\mathrm{\varrho})} \int_{0}^{t}(t-s)^{\mathrm{Q}-1} \mathbb{H}_{4}(s, \overline{\mathbb{T}}(s)) d s .
\end{aligned}
$$

Then,

$$
\begin{aligned}
\|\mathbb{S}(t)-\overline{\mathbb{S}}(t)\| \leq & \frac{1-\mathrm{\varrho}}{\beta(\mathrm{\varrho})}\left\|\mathbb{H}_{1}(t, \mathbb{S}(t))-\mathbb{H}_{1}(t, \overline{\mathbb{S}}(t))\right\| \\
& +\frac{\mathrm{\varrho}}{\beta(\mathrm{\varrho}) \Gamma(\mathrm{\varrho})} \int_{0}^{t}(t-s)^{\mathrm{\varrho}^{-1}} \| \mathbb{H}_{1}(s, \mathbb{S}(s)) \\
& -\mathbb{H}_{1}(t, \overline{\mathbb{S}}(t))\left\|d s \leq\left[\frac{1-\mathrm{\varrho}}{\beta(\mathrm{\varrho})}+\frac{1}{\beta(\mathrm{\varrho}) \Gamma(\mathrm{\varrho})}\right] \chi_{1}\right\| \mathbb{S}-\overline{\mathbb{S}} \|,
\end{aligned}
$$

which implies

$$
\left[\frac{1-\mathrm{\varrho}}{\beta(\mathrm{\varrho})} \chi_{1}+\frac{\chi_{1}}{\beta(\mathrm{\varrho}) \Gamma(\mathrm{\varrho})}-1\right]\|\mathbb{S}-\overline{\mathbb{S}}\| \geq 0 .
$$

By (33), (36) holds if $\|\mathbb{S}-\overline{\mathbb{S}}\|=0$; this implies $\mathbb{S}(t)=\overline{\mathbb{S}}(t)$. With the same procedure, for $\llbracket$, we have

$$
\begin{aligned}
\|\square(t)-\bar{\square}(t)\| \leq & \frac{1-\mathrm{\varrho}}{\beta(\mathrm{\varrho})}\left\|\mathbb{H}_{2}(t, \mathbb{\square}(t))-\mathbb{H}_{2}(t, \bar{\square}(t))\right\| \\
& +\frac{\mathrm{\varrho}}{\beta(\mathrm{\varrho}) \Gamma(\mathrm{\varrho})} \int_{0}^{t}(t-s)^{\mathrm{Q}-1} \| \mathbb{H}_{2}(s, \square(s)) \\
& -\mathbb{H}_{2}(t, \overline{\mathbb{a}}(t))\left\|d s \leq\left[\frac{1-\mathrm{\varrho}}{\beta(\mathrm{\varrho})}+\frac{1}{\beta(\mathrm{\varrho}) \Gamma(\mathrm{\varrho})}\right] \chi_{2}\right\| \square-\bar{\square} \|,
\end{aligned}
$$

which implies

$$
\left[\frac{1-\mathrm{\varrho}}{\beta(\mathrm{\varrho})} \chi_{1}+\frac{\chi_{1}}{\beta(\mathrm{\varrho}) \Gamma(\mathrm{\varrho})}-1\right]\|\mathbb{P}-\overline{\mathbb{P}}\| \geq 0 .
$$

By (33), (38) is true if $\|\mathbb{P}-\overline{\mathbb{P}}(t)\|=0$; this implies $\mathbb{P}(t)=$ $\overline{\mathbb{P}}(t)$. With the same procedure, for $\mathbb{T}$, we have

$$
\begin{aligned}
\|\mathbb{T}(t)-\overline{\mathbb{T}}(t)\| \leq & \frac{1-\mathrm{\varrho}}{\beta(\mathrm{\varrho})}\left\|\mathbb{H}_{3}(t, \mathbb{T}(t))-\mathbb{H}_{3}(t, \overline{\mathbb{T}}(t))\right\| \\
& +\frac{\mathrm{\varrho}}{\beta(\mathrm{\varrho}) \Gamma(\mathrm{\varrho})} \int_{0}^{t}(t-s)^{\mathrm{Q}-1} \| \mathbb{H}_{3}(s, \mathbb{T}(s)) \\
& -\mathbb{H}_{3}(t, \overline{\mathbb{T}}(t))\left\|d s \leq\left[\frac{1-\mathrm{\varrho}}{\beta(\mathrm{\varrho})}+\frac{1}{\beta(\mathrm{\varrho}) \Gamma(\mathrm{\varrho})}\right] \chi_{3}\right\| \mathbb{\mathbb { }}-\overline{\mathbb{T}} \|,
\end{aligned}
$$

which implies

$$
\left[\frac{1-\mathrm{\varrho}}{\beta(\mathrm{\varrho})} \chi_{4}+\frac{\chi_{4}}{\beta(\mathrm{\varrho}) \Gamma(\mathrm{\varrho})}-1\right]\|\mathbb{T}-\overline{\mathbb{T}}\| \geq 0
$$

By (33), (40) holds if $\|\mathbb{T}-\overline{\mathbb{T}}\|=0$, which implies $\mathbb{T}(t)=\overline{\mathbb{T}}$ $(t)$. Similarly, $\mathbb{T}(t)=\overline{\mathbb{T}}(t)$. Thus, the ABC-fractional order (8) has a unique solution.

\section{Hyers-Ulam Stability}

Definition 8. The integral system (23)-(26) is Hyers-Ulam stable if for $\Delta_{i}>0, i \in \mathbb{N}_{1}^{4}$, and $\gamma_{i}>0, i \in \mathbb{N}_{1}^{5}$, such that

$$
\begin{aligned}
& \left|\mathbb{S}(t)-\frac{1-\mathrm{\varrho}}{\beta(\mathrm{\varrho})} \mathbb{H}_{1}(t, \mathbb{S}(t))-\frac{\mathrm{\varrho}}{\beta(\mathrm{\varrho}) \Gamma(\mathrm{\varrho})} \int_{0}^{t}(t-s)^{\mathrm{\varrho}-1} \mathbb{H}_{1}(s, \mathbb{S}(s)) d s\right| \leq \gamma_{1}, \\
& \left|\mathrm{D}(t)-\frac{1-\mathrm{\varrho}}{\beta(\mathrm{\varrho})} \mathbb{H}_{2}(t, \mathbb{(}(t))-\frac{\mathrm{\varrho}}{\beta(\mathrm{\varrho}) \Gamma(\mathrm{\varrho})} \int_{0}^{t}(t-s)^{\mathrm{\varrho}-1} \mathbb{H}_{2}(s, \mathbb{\square}(s)) d s\right| \leq \gamma_{2}, \\
& \left|\mathbb{P}(t)-\frac{1-\mathrm{\varrho}}{\beta(\mathrm{\varrho})} \mathbb{H}_{3}(t, \mathbb{P}(t))-\frac{\mathrm{\varrho}}{\beta(\mathrm{\varrho}) \Gamma(\mathrm{\varrho})} \int_{0}^{t}(t-s)^{\mathrm{Q}-1} \mathbb{H}_{3}(s, \mathbb{P}(s)) d s\right| \leq \gamma_{3}, \\
& \left|\mathbb{T}(t)-\frac{1-\mathrm{\varrho}}{\beta(\mathrm{\varrho})} \mathbb{H}_{4}(t, \mathbb{T}(t))-\frac{\mathrm{\varrho}}{\beta(\mathrm{\varrho}) \Gamma(\mathrm{\varrho})} \int_{0}^{t}(t-s)^{\mathrm{Q}^{-1}} \mathbb{H}_{4}(s, \mathbb{T}(s)) d s\right| \leq \gamma_{4} .
\end{aligned}
$$

We have $\dot{\mathbb{S}}(t), \dot{\mathbb{I}}(t), \dot{\mathbb{P}}(t), \dot{\mathbb{T}}(t)$ which implies

$\dot{\mathbb{S}}(t)=\frac{1-\mathrm{\varrho}}{\beta(\mathrm{\varrho})} \mathbb{H}_{1}(t, \dot{\mathbb{S}}(t))+\frac{\mathrm{\varrho}}{\beta(\mathrm{\varrho}) \Gamma(\mathrm{\varrho})} \int_{0}^{t}(t-s)^{\mathrm{Q}^{-1}} \mathbb{H}_{1}(s, \dot{\mathbb{S}}(s)) d s$,

$\dot{\mathrm{d}}(t)=\frac{1-\mathrm{\varrho}}{\beta(\mathrm{\varrho})} \mathbb{H}_{2}(t, \dot{\mathrm{d}}(t))+\frac{\mathrm{\varrho}}{\beta(\mathrm{\varrho}) \Gamma(\mathrm{\varrho})} \int_{0}^{t}(t-s)^{\mathrm{Q}-1} \mathbb{H}_{2}(s, \dot{\square}(s)) d s$,

$\dot{\mathbb{P}}(t)=\frac{1-\rho}{\beta(\rho)} \mathbb{H}_{3}(t, \dot{\mathbb{P}}(t))+\frac{\rho}{\beta(\rho) \Gamma(\rho)} \int_{0}^{t}(t-s)^{\rho-1} \mathbb{H}_{3}\left(s, \dot{\square}_{H}(s)\right) d s$,

$\dot{\mathbb{T}}(t)=\frac{1-\varrho}{\beta(\mathrm{\varrho})} \mathbb{H}_{4}(t, \dot{\mathbb{T}}(t))+\frac{\mathrm{\varrho}}{\beta(\mathrm{\varrho}) \Gamma(\mathrm{\varrho})} \int_{0}^{t}(t-s)^{\mathrm{\varrho}-1} \mathbb{H}_{4}\left(s, \dot{\mathbb{I}}_{T H}(s)\right) d s$,

such that

$$
\begin{aligned}
& |\mathbb{S}(t)-\dot{\mathbb{S}}(t)| \leq \delta_{1} \gamma_{1}, \\
& |\mathbb{\square}(t)-\dot{\mathbb{\mathbb { }}}(t)| \leq \delta_{2} \gamma_{2}, \\
& |\mathbb{P}(t)-\mathbb{P}(t)| \leq \delta_{3} \gamma_{3}, \\
& |\mathbb{T}(t)-\dot{\mathbb{T}}(t)| \leq \delta_{4} \gamma_{4} .
\end{aligned}
$$

Theorem 9. Let $(\mathbb{B})$ be satisfied. Then, ABC-fractional order HCV model (8) is Hyers-Ulam stable.

Proof. By Theorem 7, the $A B C$-fractional order HCV model (8) has a unique solution, say $\mathbb{S}(t), \square(t), \mathbb{P}(t), \mathbb{T}(t)$. Let us consider $\dot{\mathbb{S}}(t), \dot{\mathbb{i}}(t), \mathbb{P}(t), \dot{\mathbb{T}}(t)$ to be another solution of (8) satisfying (23)-(26). Then, we have 


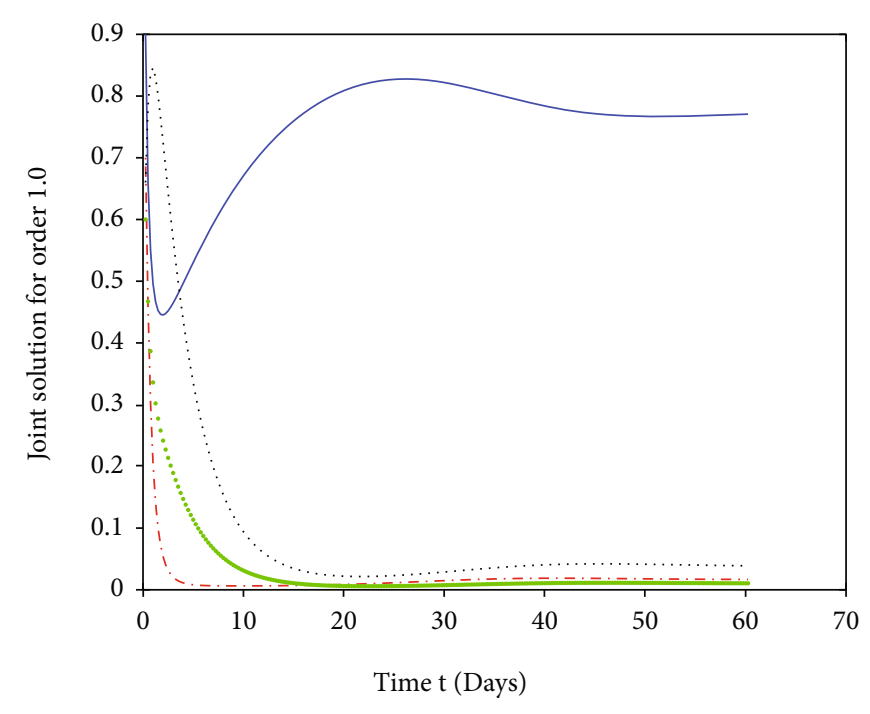



$$
\begin{aligned}
& +\frac{\mathrm{\varrho}}{\beta(\mathrm{\varrho}) \Gamma(\mathrm{\varrho})} \int_{0}^{t}(t-s)^{\mathrm{Q}-1}\left\|\mathbb{H}_{1}(s, \mathbb{S}(s))-\mathbb{H}_{1}(t, \dot{\mathbb{S}}(t))\right\| d s \\
& \leq\left[\frac{1-\mathrm{\varrho}}{\beta(\mathrm{\varrho})}+\frac{1}{\beta(\mathrm{\varrho}) \Gamma(\mathrm{\varrho})}\right] \chi_{1}\|\mathbb{S}-\dot{\mathbb{S}}\| \text {. }
\end{aligned}
$$

Taking $\gamma_{1}=\chi_{1}, \quad \Delta=(1-\mathrm{Q}) / \beta(\mathrm{Q})+\mathrm{Q} / \beta(\mathrm{\varrho}) \Gamma(\mathrm{\varrho})$, this implies

$$
\|\mathbb{S}(t)-\dot{\mathbb{S}}(t)\| \leq \gamma_{1} \Delta_{1}
$$

Similarly, for $\llbracket(t), \dot{\mathbb{\square}}(t), \mathbb{P}(t), \dot{\mathbb{P}}(t), \mathbb{T}(t), \dot{\mathbb{T}}(t)$, we have

$$
\left\{\|\llbracket(t)-\dot{\mathbb{1}}(t)\| \leq \gamma_{2} \Delta,\|\mathbb{P}(t)-\dot{\mathbb{P}}(t)\| \leq \gamma_{3} \Delta,\|\mathbb{T}(t)-\dot{\mathbb{T}}(t)\| \leq \gamma_{4} \Delta .\right.
$$

This implies that system (8) is Hyers-Ulam stable which ultimately ensures the stability of (8). This completes the proof.

\section{Numerical Scheme}

We provide the following numerical scheme by the Caputo fractional derivative:

$$
\left\{\begin{array}{l}
{ }_{0}^{A B C} \mathbb{D}_{t}^{\mathrm{Q}} \mathbb{S}(t)=\mathbb{M}_{1}(t, \mathbb{S}), \\
{ }^{A B C} \mathbb{D}_{t}^{\mathrm{e}} \mathbb{\Xi}_{T}(t)=\mathbb{M}_{2}\left(t, \mathbb{\square}_{T}\right), \\
{ }_{0}^{A B C} \mathbb{D}_{t}^{\mathrm{Q}} \mathbb{\square}_{H}(t)=\mathbb{M}_{3}\left(t, \mathbb{\square}_{H}\right), \\
{ }_{0}^{A B C} \mathbb{D}_{t}^{\mathrm{e}} \mathbb{\square}_{T H}(t)=\mathbb{M}_{4}\left(t, \mathbb{\square}_{T H}\right) .
\end{array}\right.
$$

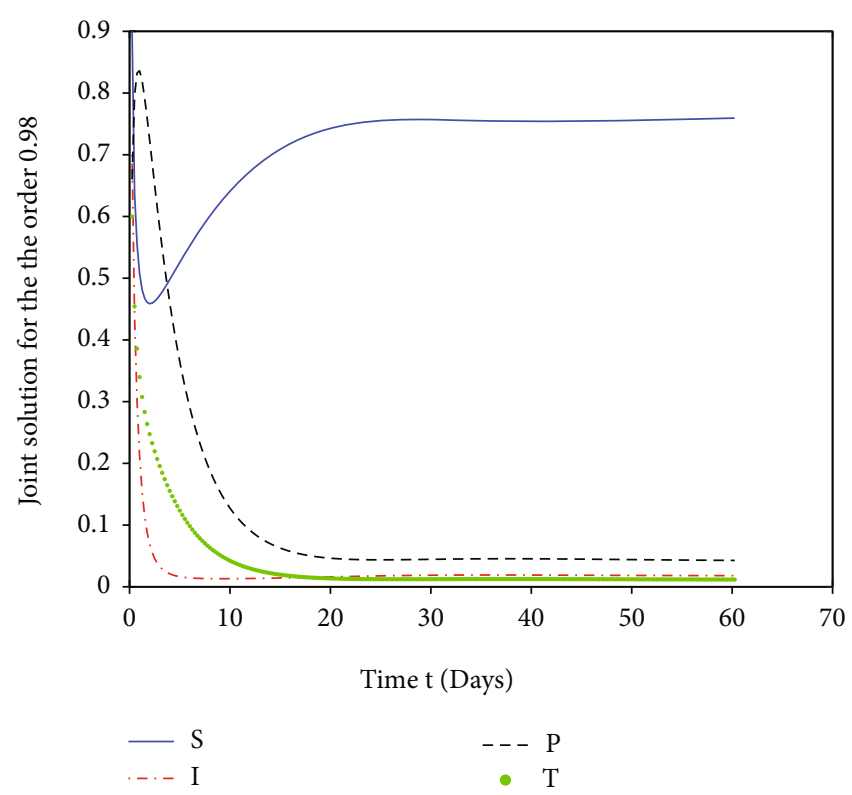

FIgURE 2: The joint solution for $\mathrm{Q}=0.98$.

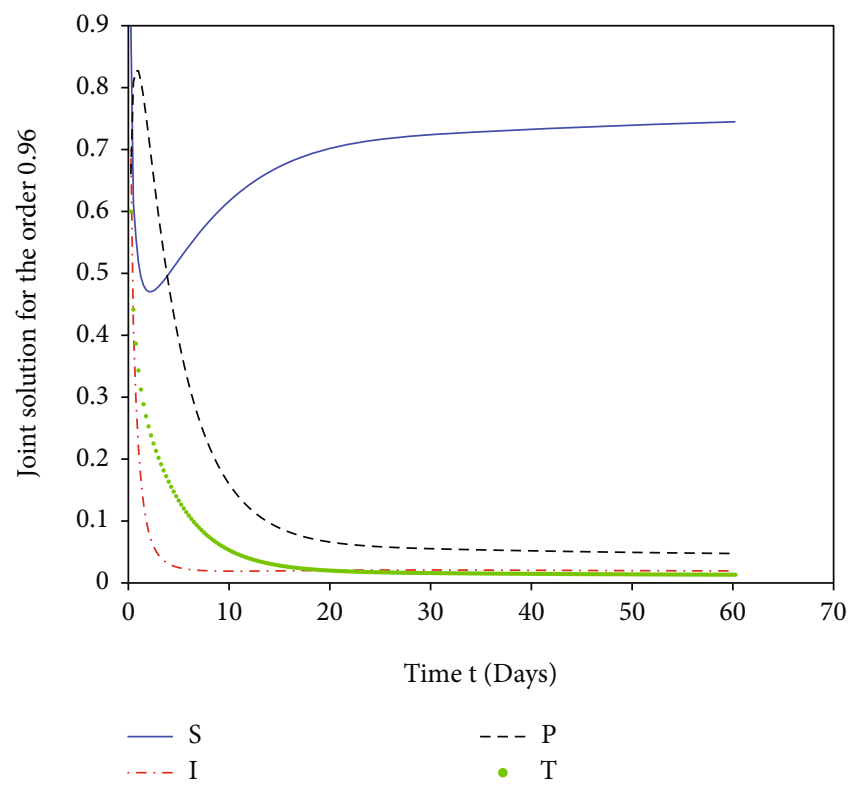

Figure 3: The joint solution for $\mathrm{Q}=0.96$.

With the help of fractional $A B$-integral operator, (48) gets the following form:

$\mathbb{S}(t)-\mathbb{S}(0)=\frac{1-\varrho}{\beta(\varrho)} \mathbb{M}_{1}(t, \mathbb{S})+\frac{\varrho}{\beta(\varrho) \Gamma(\varrho)} \int_{0}^{t}(t-s)^{\varrho^{-1}} \mathbb{M}_{1}(s, \mathbb{S}) d s$

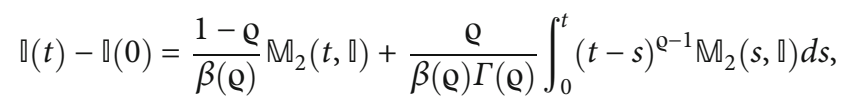




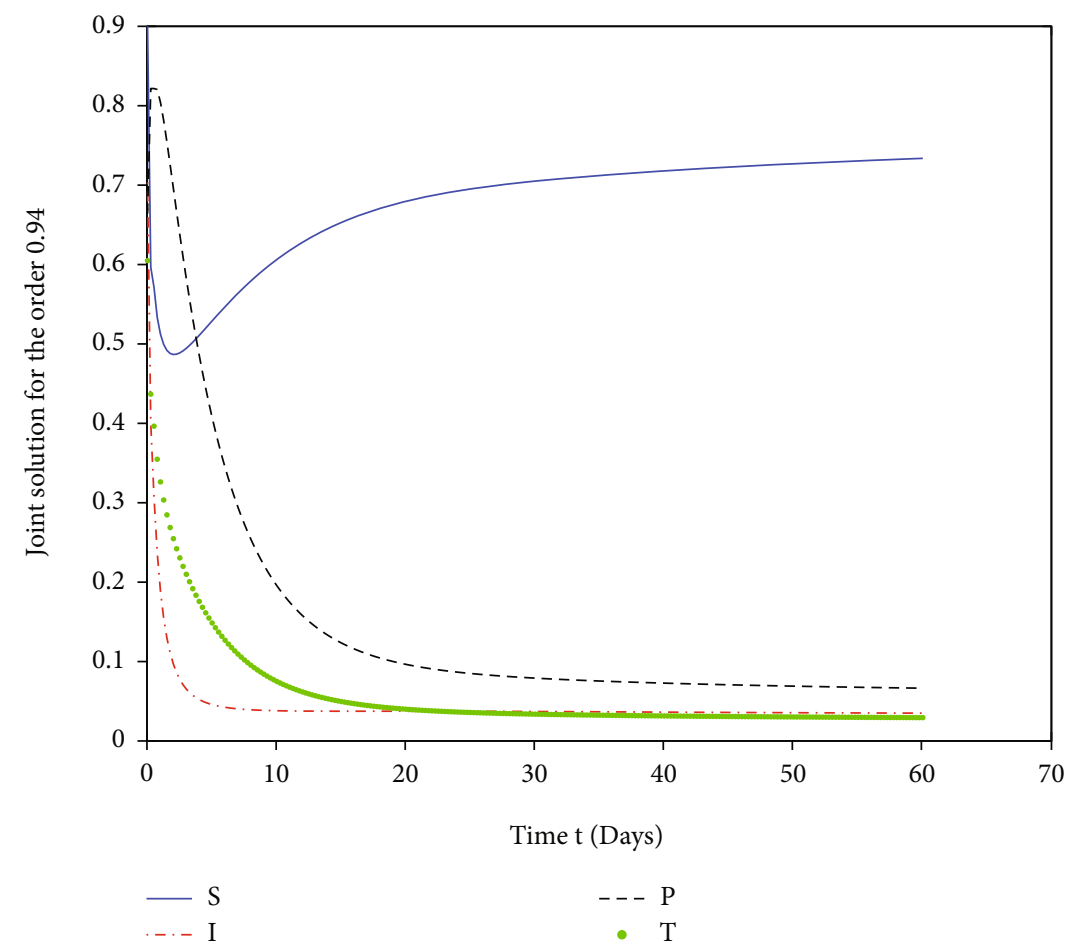

FIGURE 4: The joint solution for $\mathrm{Q}=0.94$.

$\mathbb{P}(t)-\mathbb{P}(0)=\frac{1-\varrho}{\beta(\varrho)} \mathbb{M}_{3}(t, \mathbb{P})+\frac{\varrho}{\beta(\varrho) \Gamma(\varrho)} \int_{0}^{t}(t-s)^{\mathrm{Q}-1} \mathbb{M}_{3}(s, \mathbb{P}) d s$,

$\mathbb{T}(t)-\mathbb{T}(0)=\frac{1-\varrho}{\beta(\mathrm{\varrho})} \mathbb{M}_{4}(t, \mathbb{P})+\frac{\mathrm{\varrho}}{\beta(\mathrm{\varrho}) \Gamma(\mathrm{\varrho})} \int_{0}^{t}(t-s)^{\rho-1} \mathbb{M}_{4}(s, \mathbb{T}) d s$.

By dividing the assumed interval $[0, t]$ into subintervals by the help of point $t_{n+1}$, for $n=0,1,2, \cdots$, we have

$$
\begin{aligned}
\mathbb{S}\left(t_{n+1}\right)-\mathbb{S}(0)= & \frac{1-\mathrm{\varrho}}{\beta(\mathrm{\varrho})} \mathbb{M}_{1}\left(t_{n}, \mathbb{S}\right)+\frac{\mathrm{\varrho}}{\beta(\mathrm{\varrho}) \Gamma(\mathrm{\varrho})} \sum_{k=0}^{n} \int_{t_{k}}^{t_{k+1}} \\
& \cdot\left(t_{n+1}-s\right)^{\mathrm{\varrho}-1} \mathbb{M}_{1}(s, \mathbb{S}) d s, \\
\square\left(t_{n+1}\right)-\llbracket(0)= & \frac{1-\mathrm{\varrho}}{\beta(\mathrm{\varrho})} \mathbb{M}_{2}\left(t_{n}, \mathbb{\square}\right)+\frac{\mathrm{\varrho}}{\beta(\mathrm{\varrho}) \Gamma(\mathrm{\varrho})} \sum_{k=0}^{n} \int_{t_{k}}^{t_{k+1}} \\
& \cdot\left(t_{n+1}-s\right)^{\mathrm{\varrho}-1} \mathbb{M}_{2}(s, \mathrm{\square}) d s, \\
\mathbb{P}\left(t_{n+1}\right)-\mathbb{P}(0)= & \frac{1-\mathrm{\varrho}}{\beta(\mathrm{\varrho})} \mathbb{M}_{3}\left(t_{n}, \mathbb{P}\right)+\frac{\mathrm{\varrho}}{\beta(\mathrm{\varrho}) \Gamma(\mathrm{\varrho})} \sum_{k=0}^{n} \int_{t_{k}}^{t_{k+1}} \\
& \cdot\left(t_{n+1}-s\right)^{\mathrm{\varrho}-1} \mathbb{M}_{3}(s, \mathbb{P}) d s, \\
\mathbb{T}\left(t_{n+1}\right)-\mathbb{T}(0)= & \frac{1-\mathrm{\varrho}}{\beta(\mathrm{\varrho})} \mathbb{M}_{4}\left(t_{n}, \mathbb{T}\right)+\frac{\mathrm{\varrho}}{\beta(\mathrm{\varrho}) \Gamma(\mathrm{\varrho})} \sum_{k=0}^{n} \int_{t_{k}}^{t_{k+1}} \\
& \cdot\left(t_{n+1}-s\right)^{\mathrm{\varrho}-1} \mathbb{M}_{4}(s, \mathbb{T}) d s .
\end{aligned}
$$

Now, using Lagrange's interpolation, we have

$$
\begin{aligned}
& \mathbb{S}\left(t_{n+1}\right)=\mathbb{S}(0)+\frac{1-\mathrm{\varrho}}{\beta(\mathrm{\varrho})} \mathbb{M}_{1}\left(t_{k}, \mathbb{S}\right)+\frac{\mathrm{\varrho}}{\mathbb{B}(\mathrm{\varrho})} \times \sum_{k=0}^{n} \\
& \cdot\left[\frac { h ^ { \mathrm { Q } } \mathbb { M } _ { 1 } ( t _ { k } , \mathbb { S } ) } { \Gamma ( \mathrm { \varrho } + 2 ) } \left((n+1-k)^{\mathrm{Q}}(n-k+2+\mathrm{Q})\right.\right. \\
& \left.-(n-k)^{\varrho}(n-k+2+2 \varrho)\right)-\frac{h^{\varrho} \mathbb{Z}_{1}\left(t_{k-1}, \mathbb{S}\right)}{\Gamma(\varrho+2)} \\
& \left.\times\left((n+1-k)^{\mathrm{Q}}-(n-k)^{\mathrm{Q}}(n+1-k+\mathrm{Q})\right)\right], \\
& \square\left(t_{n+1}\right)=\square(0)+\frac{1-\varrho}{\beta(\varrho)} \mathbb{M}_{2}\left(t_{k}, \square\right)+\frac{\varrho}{\mathbb{B}(\varrho)} \times \sum_{k=0}^{n} \\
& \cdot\left[\frac { h ^ { \varrho } \mathbb { M } _ { 2 } ( t _ { k } , \mathbb { \square } ) } { \Gamma ( \varrho + 2 ) } \left((n+1-k)^{\varrho}(n-k+2+\varrho)\right.\right. \\
& \left.-(n-k)^{\varrho}(n-k+2+2 \varrho)\right)-\frac{h^{\varrho} \mathbb{M}_{2}\left(t_{k-1}, \mathbb{a}\right)}{\Gamma(\varrho+2)} \\
& \left.\times\left((n-k+1)^{\varrho}-(n-k)^{\varrho}(n+1-k+\varrho)\right)\right], \\
& \mathbb{P}\left(t_{n+1}\right)=\mathbb{P}(0)+\frac{1-\varrho}{\beta(\varrho)} \mathbb{M}_{3}\left(t_{k}, \mathbb{P}\right)+\frac{\varrho}{\mathbb{B}(\varrho)} \times \sum_{k=0}^{n} \\
& \text {. }\left[\frac { h ^ { \mathrm { Q } } \mathbb { M } _ { 3 } ( t _ { k } , \mathbb { 0 } ) } { \Gamma ( \mathrm { Q } + 2 ) } \left((n+1-k)^{\mathrm{Q}}(n-k+2+\mathrm{Q})\right.\right. \\
& \left.-(n-k)^{\mathrm{e}}(n-k+2+2 \mathrm{\varrho})\right)-\frac{h^{\complement} \mathbb{Z}_{3}\left(t_{k-1}, \mathbb{1}_{H}\right)}{\Gamma(\mathrm{\varrho}+2)} \\
& \left.\times\left((n+1-k)^{\varrho}-(n-k)^{\varrho}(n+1-k+\varrho)\right)\right],
\end{aligned}
$$


TABLE 1: Shows the parameter values given in Equation (8). The time units are taken in years.

\begin{tabular}{lccc}
\hline Parameter & Description & Value & Reference \\
\hline$\mu$ & Rate of natural death & 0.09 & Assumed \\
$\lambda$ & Force of infection & 0.5 & {$[12]$} \\
$\psi$ & Rate of susceptibility of recovered & 0.02 & 0.7 \\
$\sigma$ & Rate of recovery from acute infection & 0.035 & {$[11-13]$} \\
$\varepsilon$ & Rate of progression to chronic infection & 0.50 & {$[12]$} \\
$d$ & Rate of death due to acute infection & 0.012 & {$[12]$} \\
$\rho$ & Rate of treatment failure of chronically infected & 0.34 & {$[12]$} \\
$\delta$ & Rate of recovery from chronic infection & 0.67 \\
\hline
\end{tabular}

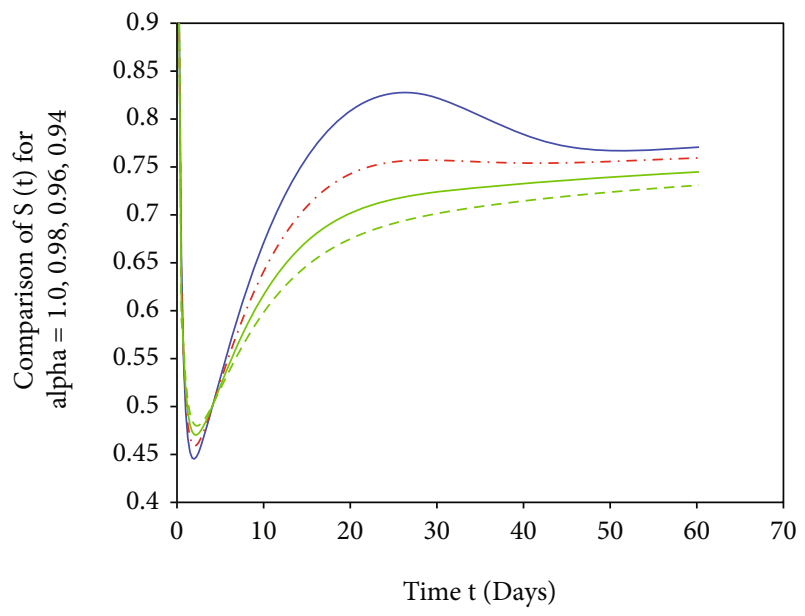

- $\mathrm{S}(\mathrm{t})$ at alpha $=1.0 \quad-\mathrm{S}(\mathrm{t})$ at alpha $=0.96$

FIgURE 5: The susceptible class $\mathbb{S}(t)$ for $\varrho=1.0, \quad 0.98, \quad 0.96, \quad 0.94$.

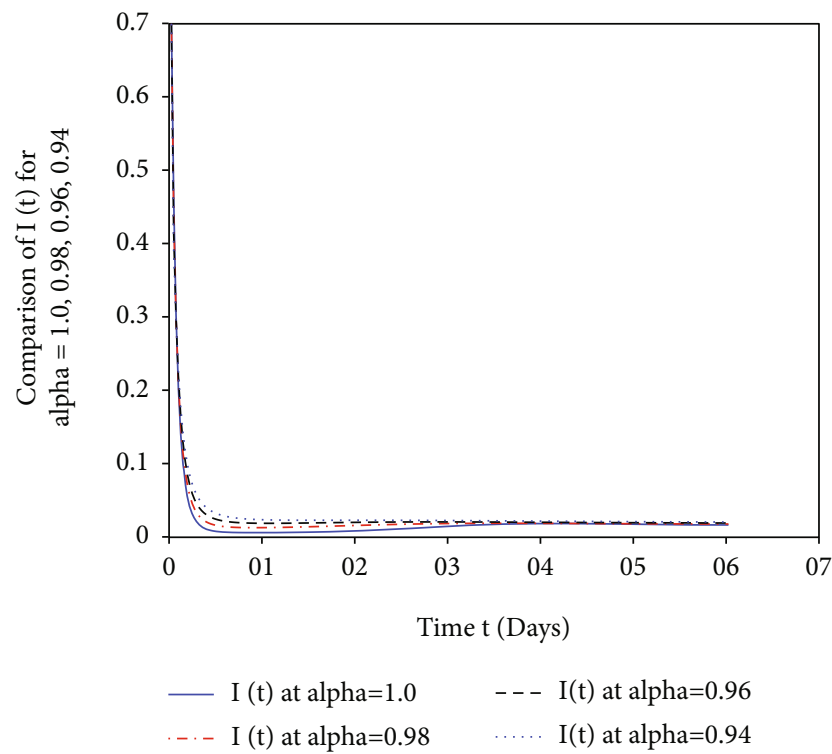

Figure 6: The infected class $\llbracket(t)$ for $\mathrm{Q}=1.0,0.98,0.96,0.94$.

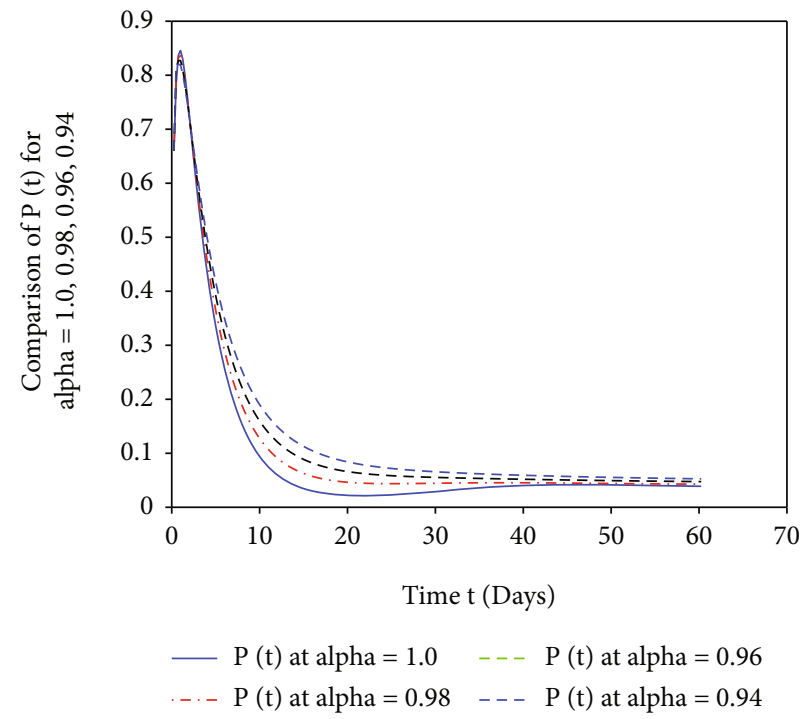

FIgure 7: The chronically infected $P(t)$ for $\mathrm{Q}=1.0,0.98,0.96,0.94$.

$$
\begin{aligned}
\mathbb{T}\left(t_{n+1}\right)= & \mathbb{T}(0)+\frac{1-\varrho}{\beta(\varrho)} \mathbb{M}_{4}\left(t_{k}, \mathbb{T}\right)+\frac{\varrho}{\mathbb{B}(\varrho)} \times \sum_{k=0}^{n} \\
& \cdot\left[\frac { h ^ { \varrho } \mathbb { M } _ { 4 } ( t _ { k } , \mathbb { T } ) } { \Gamma ( \varrho + 2 ) } \left((n+1-k)^{\varrho}(n-k+2+\varrho)\right.\right. \\
& \left.-(n-k)^{\varrho}(n-k+2+2 \mathrm{\varrho})\right)-\frac{h^{\varrho} \mathbb{M}_{4}\left(t_{k-1}, \mathbb{Q}_{T H}\right)}{\Gamma(\varrho+2)} \\
& \left.\times\left((n+1-k)^{\varrho+1}-(n-k)^{\varrho}(n+1-k+\varrho)\right)\right] .
\end{aligned}
$$

\section{Computational Results}

Here, we present some computational results based on the parametric values defined in [12]. We consider the initial values $\mathbb{S}_{0}=0.90, \mathbb{\square}_{0}=0.70, \mathbb{P}_{0}=0.66, \mathbb{\mathbb { T }}_{0}=0.60$, and the parametric values given by $\beta=1.30, \mu=0.09, \chi=0.5, \psi=$ $0.5, \sigma=0.5, \varepsilon=0.7, d=0.035, \rho=0.50, \delta=0.012, \wp=0.34, \theta$ $=0.67$. We get the same behavior of the fractional order model as for the integer order. 


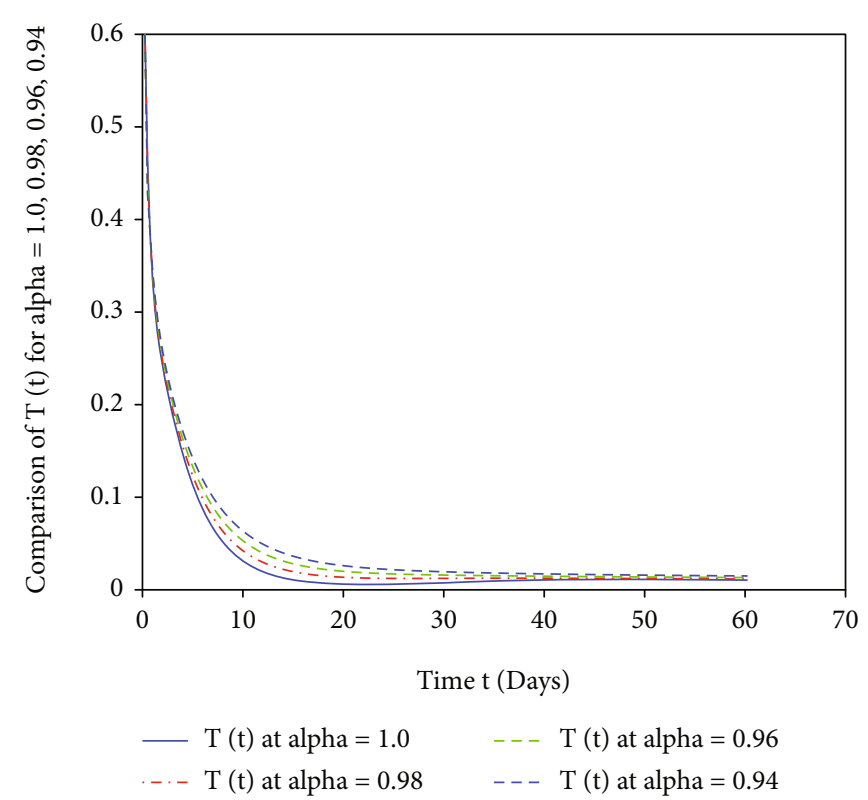

FIGURE 8: Chronically infected treated class $T(t)$ for $\mathrm{Q}=1.0,0.98$, $0.96,0.94$.

Figures 1-4 represent the numerical solution of the model for the orders $\varrho=1.0,0.98,0.96,0.94$ and using Table 1 values, respectively. Figure 5 represents the susceptible class for the orders $\mathrm{Q}=1.0,0.98,0.96,0.94$ and using Table 1 values, which are increasing with the passage of time and get stability after 60 days of the treatment. The infection class is given in Figure 6, for the fractional orders $\mathrm{Q}=1.0$, $0.98,0.96,0.94$ and using Table 1 values, it has been observed that with the passage of time, the infection is decreased to a certain limit. Figure 7 shows the chronic infection class for $\varrho=1.0,0.98,0.96,0.94$, and using Table 1 values, finally the chronically infected treated class for $\mathrm{Q}=1.0,0.98,0.96,0.94$ is given in Figure 8. This numerical analysis of the fractional order model ensures that the fractional order model is more informative and has the same behavior as the classical model.

\section{Conclusions}

In this article, we have given a mathematical fractional order Hepatitis $\mathrm{C}$ virus (HCV) spread model for an analytical and numerical study. The model is a fractional order extension of the classical model. The paper includes the existence, uniqueness, Hyers-Ulam stability, and a numerical scheme for the computational results. Our numerical results are based on the Lagrange polynomial interpolation. On the basis of the numerical scheme, we have given graphical explanation of the model and its subclasses. For details, Figures 1-4 represent the numerical solution of the model with the fractional orders $\mathrm{Q}=1.0,0.98,0.96,0.94$, respectively. Figure 5 represents the susceptible class for the orders $\mathrm{Q}=1.0,0.98,0.96,0.94$ which are increasing with the passage of time and get stability after 60 days of treatment. The infection class is given in Figure 6 for the fractional orders $\mathrm{Q}=1.0,0.98,0.96,0.94$, and it has been observed that with the passage of time the infection is decreased to a certain limit. Figure 7 shows the chronic infection class for $\mathrm{Q}=1.0,0.98,0.96,0.94$, and finally, the chronically infected treated class for $\mathrm{Q}=1.0,0.98,0.96,0.94$ is given in Figure 8 . Our numerical analysis of the fractional order model ensures that the fractional order model is more informative and has the same behavior as the classical model.

\section{Data Availability}

The data used to support the findings of this study are available from the corresponding author upon request.

\section{Conflicts of Interest}

The authors declare no conflict of interest.

\section{Authors' Contributions}

All authors contributed equally in writing this article. All authors read and approved the final manuscript.

\section{Acknowledgments}

This project was funded by the Deanship of Scientific Research (DSR) at King Abdulaziz University, Jeddah, under grant no. (G: 69-130-1442). The authors, therefore, acknowledge with thanks DSR for technical and financial support.

\section{References}

[1] Centers for Disease Control and Prevention, Viral Hepatitis, Centers for Disease Control and Prevention website, 2016.

[2] K. J. Ryan and C. G. Ray, Medical Microbiology, vol. 4, McGraw Hill, 2004.

[3] A. Maheshwari and P. J. Thuluvath, "Management of acute hepatitis C," Clinics in Liver Disease, vol. 14, no. 1, pp. 169176, 2010.

[4] C. Castillo-Chavez and B. Song, "Dynamical models of tuberculosis and their applications," Mathematical Biosciences of Engineering, vol. 1, no. 2, pp. 361-404, 2004.

[5] M. A. Khan, Z. Hammouch, and D. Baleanu, "Modeling the dynamics of hepatitis Eviathe Caputo-Fabrizio derivative," Mathematical Modelling of Natural Phenomena, vol. 14, no. 3, pp. 311-319, 2019.

[6] S. Uçar, E. Uçar, N. Özdemir, and Z. Hammouch, "Mathematical analysis and numerical simulation for a smoking model with Atangana-Baleanu derivative," Chaos, Solitons \& Fractals, vol. 118, pp. 300-306, 2019.

[7] F. Evirgen, S. Uçar, and N. Özdemi, "System analysis of HIV infection model withCD4+Tunder non-singular kernel derivative," Applied Mathematics and Nonlinear Sciences, vol. 5, no. 1, pp. 139-146, 2020.

[8] J. Danane, K. Allali, Z. Hammouch, and K. S. Nisar, "Mathematical analysis and simulation of a stochastic COVID-19 Levy jump model with isolation strategy," Results in Physics, vol. 23, p. 103994, 2021.

[9] M. Zamir, F. Nadeem, T. Abdeljawad, and Z. Hammouch, "Threshold condition and non pharmaceutical interventions's control strategies for elimination of COVID-19," Results in Physics, vol. 20, p. 103698, 2021. 
[10] D. Clayton and M. Hills, Statistical Models in Epidemiology, OUP Oxford, 2013.

[11] S. L. Chen and T. R. Morgan, "The natural history of hepatitis $C$ virus (HCV) infection," International Journal of Medical Sciences, vol. 3, no. 2, pp. 47-52, 2006.

[12] R. Shi and Y. Cui, "Global analysis of a mathematical model for Hepatitis C virus transmissions," Virus Research, vol. 217, pp. 8-17, 2016.

[13] J. Khodaei-Mehr, S. Tangestanizadeh, R. Vatankhah, and M. Sharifi, "Optimal neuro-fuzzy control of hepatitis C virus integrated by genetic algorithm," IET Systems Biology, vol. 12, no. 4, pp. 154-161, 2018.

[14] G. Blé, L. Esteva, and A. Peregrino, "Global analysis of a mathematical model for hepatitis $\mathrm{C}$ considering the host immune system," Journal of Mathematical Analysis and Applications, vol. 461, no. 2, pp. 1378-1390, 2018.

[15] A. Atangana and I. Koca, "Chaos in a simple nonlinear system with Atangana-Baleanu derivatives with fractional order," Chaos, Solitons \& Fractals, vol. 89, pp. 447-454, 2016.

[16] D. Baleanu, R. P. Agarwal, R. K. Parmar, M. Alqurashi, and S. Salahshour, "Extension of the fractional derivative operator of the Riemann-Liouville," Journal Nonlinear Sciences Applications, vol. 10, no. 6, pp. 2914-2924, 2017.

[17] T. Abdeljawad and D. Baleanu, "Discrete fractional differences with nonsingular discrete Mittag-Leffler kernels," Advances in Difference Equations, vol. 2016, no. 1, 18 pages, 2016.

[18] H. Khan, W. Chen, and H. Sun, "Analysis of positive solution and Hyers-Ulam stability for a class of singular fractional differential equations with p-Laplacian in Banach space," Mathematical Methods in the Applied Sciences, vol. 41, no. 9, pp. 3430-3440, 2018.

[19] A. Atangana and R. T. Alqahtani, "Modelling the spread of river blindness disease via the caputo fractional derivative and the beta-derivative," Entropy, vol. 18, no. 2, p. 40, 2016.

[20] J. F. Gómez-Aguilar, M. G. López-López, V. M. AlvaradozMartinez, D. Baleanu, and H. Khan, "Chaos in a cancer model via fractional derivatives with exponential decay and MittagLeffler law," Entropy, vol. 19, no. 12, p. 681, 2017.

[21] K. Shah and S. Bushnaq, "Numerical treatment of fractional endemic disease model via Laplace Adomian decomposition method," Journal of Science and Arts, vol. 17, no. 2, p. 257, 2017.

[22] M. Arfan, K. Shah, T. Abdeljawad, N. Mlaiki, and A. Ullah, “A Caputo power law model predicting the spread of the COVID19 outbreak in Pakistan," Alexandria Engineering Journal, vol. 60, no. 1, pp. 447-456, 2021.

[23] T. Abdeljawad, Q. M. Al-Mdallal, and F. Jarad, "Fractional logistic models in the frame of fractional operators generated by conformable derivatives," Chaos, Solitons \& Fractals, vol. 119, pp. 94-101, 2019.

[24] N. Sene, "SIR epidemic model with Mittag-Leffler fractional derivative," Chaos, Solitons \& Fractals, vol. 137, p. 109833, 2020.

[25] B. Ghanbari and C. Cattani, "On fractional predator and prey models with mutualistic predation including non-local and nonsingular kernels," Chaos, Solitons \& Fractals, vol. 136, p. $109823,2020$.
[26] K. M. Owolabi, "Modelling and simulation of a dynamical system with the Atangana-Baleanu fractional derivative," The European Physical Journal Plus, vol. 133, no. 1, pp. 1-13, 2018.

[27] A. Atangana and D. Baleanu, "New fractional derivatives with nonlocal and non-singular kernel: theory and application to heat transfer model," 2016, https://arxiv.org/abs/1602.03408. 